\title{
Real-world safety and efficacy of nivolumab for $\geq 2$ nd line treatment of metastatic renal cell carcinoma: A retrospective cohort study in Croatia, Hungary, and Malta
}

\author{
Eduard VRDOLJAK ${ }^{1, \star}$, Claude MAGRI ${ }^{2}$, Marija GAMULIN ${ }^{3}$, Lidija BOŠKOVIĆ ${ }^{1}$, Tomislav OMRČEN ${ }^{1}$, Žarko BAJIĆ $^{4}$, Tamas DIENES ${ }^{5}$, Lajos GECZI ${ }^{5}$ \\ ${ }^{1}$ Department of Oncology, University Hospital Split, School of Medicine, University of Split, Split, Croatia; ${ }^{2}$ Mater Dei Hospital, Triq Dun Karm, \\ L-Imsida, Malta; ${ }^{3}$ Department of Oncology, University Hospital Center Zagreb, University of Zagreb School of Medicine, Zagreb, Croatia; \\ ${ }^{4}$ Research unit "Dr. Mirko Grmek", Psychiatric Hospital Sveti Ivan, Zagreb; Croatia; ${ }^{5}$ National Institute of Oncology, Budapest, Hungary
}

${ }^{*}$ Correspondence: edo.vrdoljak@gmail.com

Received May 12, 2020 / Accepted August 12, 2020

\begin{abstract}
The objective of our study was to assess the real-world safety and efficacy of nivolumab in the second- or later-line treatment of metastatic renal cell carcinoma (mRCC). We conducted a multicenter, retrospective, observational study of realworld data from patients who were treated with nivolumab under a patient expanded access program from 2015 to 2017 in Croatia, Hungary, and Malta. The primary safety endpoint was the discontinuation of therapy because of adverse events. The primary efficacy endpoint was overall survival (OS). We collected data from 87 patients with a median (interquartile range (IQR)) age of 63 (57-68) years, and $21 \%$ were females. The median (IQR) follow-up was 11 (5-31) months. Treatment was discontinued because of toxicity in 4 (5\%) patients. Four (5\%) patients experienced treatment-related adverse events of grade 3 or 4 . The OS was 18.0 (95\% CI: 11.0 to 28.6) months, and the PFS was 8.5 (95\% CI: 4.9 to 12.1) months. Our study indicated a good safety and efficacy profile of nivolumab in the second- or later-line treatment of mRCC patients in a realworld clinical practice environment, which is comparable with the findings of the registrational trial.
\end{abstract}

Key words: metastatic renal carcinoma, PD-1 immune checkpoint inhibitor, nivolumab, immunotherapy

An understanding of the mechanisms involved in the carcinogenesis of clear cell renal carcinoma has led to the development of targeted therapies directed against vascular endothelial growth factor [1-7]. With optimal utilization of such agents throughout multiple lines of therapies, the median overall survival time of metastatic renal cell carcinoma (mRCC) patients more than doubled: from 12 months in the cytokine era to 30 months in tyrosine kinase inhibitor (TKI) era [1-8]. Unfortunately, despite an observed benefit associated with TKIs in terms of progression-free survival (PFS) and overall survival (OS), almost all patients with mRCC eventually experience disease progression and ultimately die, highlighting the need for more effective treatment options. Multiple possible immune resistance mechanisms have been described in tumor development $[9,10]$. The most promising mechanism from a treatment point of view is the inhibition of immune checkpoints [11]. The recent understanding of these immune mechanisms has resulted in the generation of many antibodies directed against immune checkpoint receptors that are already widely used in therapy for a variety of cancers in everyday practice, mRCC included [12]. Nivolumab is a human immunoglobulin G4 programmed death-1 immune checkpoint inhibitor antibody that selectively blocks the interaction between PD-1 and its ligands PD-L1 and PD-L2, a mechanism that usually leads to the inhibition of cellular immune response [13]. By inhibiting this inhibition, nivolumab can enhance antitumor T-cell activity [14]. Based on the practice-changing results of a randomized phase III study (CheckMate 025), nivolumab is now widely used as a standard second- or later-line treatment option (after first-line TKI therapy) in the treatment of mRCC [15]. Expanded-access programs generally apply less stringent entry criteria than clinical trials and allow patients who have no access to, or who are ineligible for, clinical trials the opportunity to receive a new drug therapy prior to its approval. The findings from expanded-access programs complement those of regular clinical trials by providing insight into real-world treatment patterns, safety, and effectiveness in a broad spectrum of community-based cancer patients, including those with poor prognosis. This retro- 
spective analysis of the nivolumab expanded access program data examines treatment outcomes among study participants in Croatia, Hungary, and Malta, countries with some of the highest rates of RCC in the world, as well as historically inferior oncological outcomes and limited second- and third-line treatment options compared with those in other parts of Europe [16-19].

\section{Patients and methods}

Study design. We conducted a multicenter, retrospective, observational study of real-world data of patients who were treated with nivolumab in an expanded-access program from November 11, 2015 to January 2, 2017 at the Department of Oncology, Clinical Hospital Center Split, Split, Croatia; Department of Oncology, University Hospital Center Zagreb, Zagreb, Croatia; National Institute of Oncology, Budapest, Hungary; and Mater Dei Hospital, Triq Dun Karm, L-Imsida, Malta. The study protocol was evaluated and approved by the ethics committees of all participating institutions. Patients who were alive at the time of data collection signed the informed consent forms. Before the analysis, we anonymized all the data and concealed the patients' identities to everyone but the patients' oncologists. We performed the study in accordance with the World Medical Association Declaration of Helsinki of 1975, as revised in 2013, and the International Conference on Harmonization Guidelines on Good Clinical Practice [20]. The study was sponsored by Bristol-Myers Squibb. We did not pre-register the protocol.

Study population. The inclusion criteria were age $\geq 18$ years, histologically confirmed advanced or metastatic RCC with a clear cell component, Eastern Cooperative Oncology Group (ECOG) performance status (PS) $\leq 2$, and treatment with at least one prior anti-angiogenic therapy regimen (including but not limited to, sunitinib, sorafenib, pazopanib, axitinib, tivozanib, and bevacizumab) in the advanced or metastatic setting. Prior cytokine therapy (e.g. IL-2, IFN-a), vaccine therapy, or treatment with cytotoxic was also allowed. Patients had to have measurable disease by CT or MRI per the RECIST 1.1 criteria; radiographic tumor assessments performed within 28 days of the first dose of the program drug. Prior targeted therapy had to be completed at least 4 weeks prior to the program drug administration, and all adverse events had to have either returned to the baseline condition or stabilized. Prior radiotherapy or radiosurgery had to be completed at least 2 weeks prior to the first dose of the program drug.

Pre-treatment laboratory values had to meet the following criteria and had to be obtained prior to the commencement of treatment: $\mathrm{WBC} \geq 2000 / \mu \mathrm{l}$, neutrophils $\geq 1500 / \mu$ l, platelets $\geq 100 \times 10^{3} / \mu \mathrm{l}$, hemoglobin $\geq 9.0 \mathrm{~g} / \mathrm{dl}$, serum creatinine $\leq 1.5 \times \mathrm{ULN}$ or $\mathrm{CrCL}>40 \mathrm{ml} / \mathrm{minute}$ [(using Cockcroft/Gault formula $)$, female $\mathrm{CrCl}=((140$-age in years $) \times$ weight in $\mathrm{kg} \times$ $0.85) \div(72 \times$ serum creatinine in $\mathrm{mg} / \mathrm{dl})$, male $\mathrm{CrCl}=((140-$ age in years $) \times$ weight in $\mathrm{kg} \times 1.00) \div(72 \times$ serum creati- nine in $\mathrm{mg} / \mathrm{dl}$ ), AST $\leq 3 \times \mathrm{ULN}$, ALT $\leq 3 \times \mathrm{ULN}$, total bilirubin $\leq 1.5 \times$ ULN (except patients with Gilbert Syndrome who can have total bilirubin $<3.0 \mathrm{mg} / \mathrm{dl}$ ).

The exclusion criteria were ECOG performance status $\geq 3$; life expectancy of $<6$ weeks; active brain or leptomeningeal metastasis; carcinomatous meningitis; ocular melanoma; known or suspected autoimmune disease; prior therapy with anti-PD-1, anti-PD-L1, anti-PD-L2, anti-CT137, or anti-CTLA-4 antibodies including ipilimumab or any other antibody or drug specifically targeting $\mathrm{T}$ cell co-stimulation or checkpoint pathways; prior treatment in any nivolumab trial, including prior treatment on either arm of nivolumab studies CA209057 or CA209026; interstitial lung disease that was symptomatic or may interfere with the detection or management of suspected drug-related pulmonary toxicity; other active malignancy requiring concurrent intervention; known alcohol or drug abuse; known history of testing positive for human immunodeficiency virus (HIV) or known acquired immunodeficiency syndrome (AIDS); history of severe hypersensitivity reactions to other monoclonal antibodies; history of allergy or intolerance to program drug components or polysorbate-80-containing infusions; pregnancy and breastfeeding; and prior malignancy active within the previous three years except for locally curable cancers that have been apparently cured, such as basal or squamous cell skin cancer, superficial bladder cancer, or carcinoma in situ of the prostate, cervix or breast.

Sample type and required sample size. We did not select the sample but rather included the total targeted population. Therefore, we did not calculate the required sample size in advance.

Endpoints. The primary safety endpoint was the discontinuation of therapy because of adverse events. The secondary safety endpoints were incidence of grade 3 or 4 treatmentrelated and immune-mediated adverse events according to the Common Terminology Criteria for Adverse Events v4.0. The primary efficacy endpoint was overall survival (OS). The secondary efficacy endpoints were progression-free survival (PFS), best response, overall response rate (ORR) defined as a partial or complete response, disease control rate (DCR) defined as a partial or complete response or the stabilization of disease, time from the introduction of nivolumab to response in months (TTR), and the duration of response (DOR) in months.

Treatment. Nivolumab (Opdivo, Bristol-Myers Squibb) was administered at a dosage of $3 \mathrm{mg} / \mathrm{kg}$ body weight via 60-minute intravenous infusions every two weeks for a maximum of 24 months or until unacceptable toxicity, disease progression, or withdrawal of informed consent occurred.

Potential confounders. In the exploratory analysis of the association of immune-mediated adverse events with the efficacy endpoints, we controlled for the possible confounding effect of the time-dependent covariate: number of nivolumab cycles. We controlled for the effects of age, sex, number of metastatic sites, ECOG performance status at the 
start of treatment with nivolumab, time from diagnosis to the introduction of nivolumab, concomitant radiotherapy, and the treatment after the discontinuation of nivolumab. Data were obtained from the institutions' medical databases.

Statistical analysis. We conducted the analysis using the data from all patients who received at least one dose of nivolumab. We estimated median TTR, DOR, PFS, and OS with $95 \%$ confidence intervals (CIs) using the Kaplan-Meyer method. In the exploratory multivariable analysis of the association of PFS and OS with the immune-mediated and treatment-related adverse events, we controlled for potential confounders using the Cox proportional hazard regression, using the Efron method for handling ties. Before this analysis and before the analysis of the effect of the subsequent therapy after the discontinuation of nivolumab, we tested the proportional hazard assumption through the inspection of log-log survival plots, Kaplan-Meier observed survival curves and the Cox regression prediction curves, and tested the slope of a generalized linear regression of the scaled Schoenfeld residuals on log-time. We set two-tailed statistical significance at $\mathrm{p}<0.05$ and calculated all confidence intervals (CIs) at the $95 \%$ level. We controlled the false positive rate using the Benjamini-Hochberg procedure with the false discovery rate set in advance at $<10 \%$. We performed statistical data analysis using StataCorp 2019 (Stata Statistical Software: Release 16. College Station, TX: StataCorp LLC).

\section{Results}

We collected data from 87 patients treated in four hospitals in Croatia, Hungary, and Malta. The patients' median (IQR) age was 63 (57-68) years, and $18(21 \%)$ were females (Table 1). At the end of follow-up, 27 (31\%) patients were alive with no progression of the disease and were censored. The median (IQR) follow-up time was 11 (5-31) months, and the median (IQR) duration of therapy with nivolumab was 7 (3-15) months.

Treatment was discontinued because of toxicity in $4 / 82$ (5\%; $95 \%$ CI: $1 \%$ to $12 \%$ ) patients, while 37 (43\%; $95 \%$ CI: $32 \%$ to $54 \%$ ) patients experienced treatment-related adverse events of any grade, and 4 (5\%; $95 \%$ CI: $0 \%$ to $10 \%$ ) patients experienced adverse events of grade 3 or 4 (Table 2). Treatment-related and immune-mediated adverse events were experienced by 22 (25\%; $95 \%$ CI: $17 \%$ to $35 \%)$ patients, and 4 (5\%; $95 \%$ CI: $0 \%$ to $10 \%)$ patients experienced immunerelated adverse events of grade 3 or 4 . We did not record any grade 5 adverse events.

OS was 18.0 (95\% CI: 11.0 to 28.6) months, and PFS was 8.5 (95\% CI: 4.9 to 12.1 ) months (Figure 1). Patients with therapy after the discontinuation of nivolumab $(n=19)$ had significantly lower HR for death (HR $=0.33$; 95\% CI: $0.15-0.73)$ compared to patients with no subsequent therapy $(\mathrm{n}=61)$. Proportional hazard assumption of the Cox regression was satisfied for these two groups. Their median $(95 \%$ CI:) OS was 27.3 (10.5-not reached) months compared
Table 1. Characteristics of patients and treatment $(n=87)$.

\begin{tabular}{|c|c|c|}
\hline & n & $(\%)$ \\
\hline Age at nivolumab initiation (years), median (IQR) & 63 & $(57-68)$ \\
\hline \multicolumn{3}{|l|}{ Sex } \\
\hline males & 69 & (79) \\
\hline females & 18 & $(21)$ \\
\hline \multicolumn{3}{|l|}{$\begin{array}{l}\text { Number of prior systemic therapies for the metastatic } \\
\text { disease }\end{array}$} \\
\hline 1 & 78 & (90) \\
\hline 2 & 9 & $(10)$ \\
\hline \multicolumn{3}{|l|}{ Prior systemic therapy for the metastatic disease } \\
\hline sunitinib & 29 & $(34)$ \\
\hline everolimus & 16 & (19) \\
\hline axitinib & 12 & (14) \\
\hline pazopanib & 9 & (10) \\
\hline sorafenib & 8 & (9) \\
\hline chemotherapy & 6 & $(7)$ \\
\hline chemoradiotherapy & 3 & $(5)$ \\
\hline cabozantinib & 2 & $(2)$ \\
\hline \multicolumn{3}{|l|}{ Metastatic sites } \\
\hline lung & 70 & $(80)$ \\
\hline mediastinal lymph nodes & 40 & $(46)$ \\
\hline bone & 40 & $(46)$ \\
\hline other sites & 34 & $(39)$ \\
\hline liver & 19 & $(22)$ \\
\hline abdominal lymph nodes & 19 & $(22)$ \\
\hline pleural effusion & 18 & $(21)$ \\
\hline pleural metastasis & 10 & (11) \\
\hline brain & 7 & $(8)$ \\
\hline Number of metastatic sites, median (IQR) & 3 & $(2-4)$ \\
\hline \multicolumn{3}{|l|}{ ECOG performance status ${ }^{a}$} \\
\hline 0 & 35 & (47) \\
\hline 1 & 30 & $(41)$ \\
\hline 2 & 9 & (12) \\
\hline $\begin{array}{l}\text { Time from diagnosis to introduction of } \\
\text { nivolumab (years), median (IQR) }\end{array}$ & 3 & $(2-4)$ \\
\hline Follow-up (months), median (IQR) & 11 & $(5-31)$ \\
\hline $\begin{array}{l}\text { Duration of therapy with nivolumab } \\
\text { (months), median (IQR) }\end{array}$ & 7 & $(3-15)$ \\
\hline Number of cycles, median (IQR) ${ }^{a}$ & 15 & $(8-29)$ \\
\hline \multicolumn{3}{|l|}{ Concomitant therapy } \\
\hline antihypertensives $^{\mathrm{a}}$ & 44 & (51) \\
\hline proton pump inhibitors & 32 & (37) \\
\hline bisphosphonates $^{\mathrm{a}}$ & 28 & (33) \\
\hline radiotherapy ${ }^{\mathrm{a}}$ & 25 & (29) \\
\hline antibiotics $^{\mathrm{a}}$ & 24 & (28) \\
\hline corticosteroids & 20 & (24) \\
\hline acetylsalicylic acid & 6 & (7) \\
\hline Therapy after nivolumab & 19 & $(24)$ \\
\hline
\end{tabular}

Data are presented as number (percentage) of patients if not stated otherwise, abbreviation: IQR-interquartile range; ${ }^{a}$ Data were missing for ECOG performance status in 13 (15\%) paitents; for duration of therapy with nivolumab in $6(7 \%)$ patients; for number of cycles in $7(8 \%)$ patients; for antihypertensives in 1 (1\%) patient; for bisphosphonates, radiotherapy and antibiotics in $2(2 \%)$ patients 


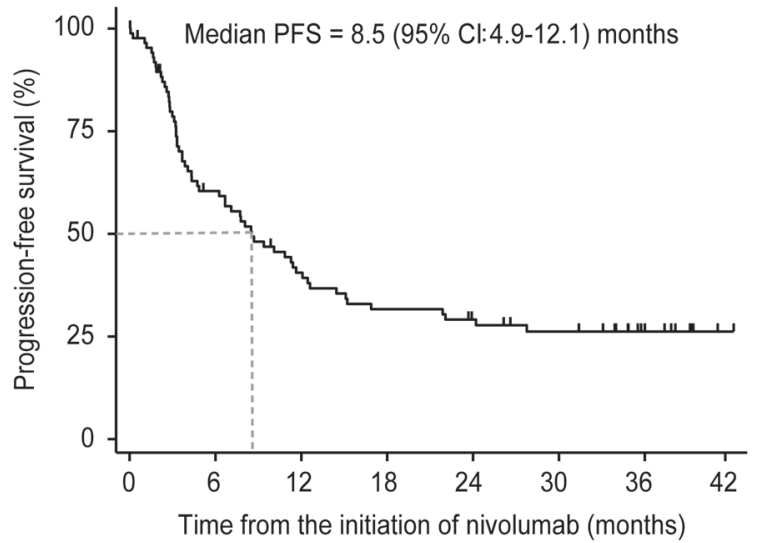

Number at risk

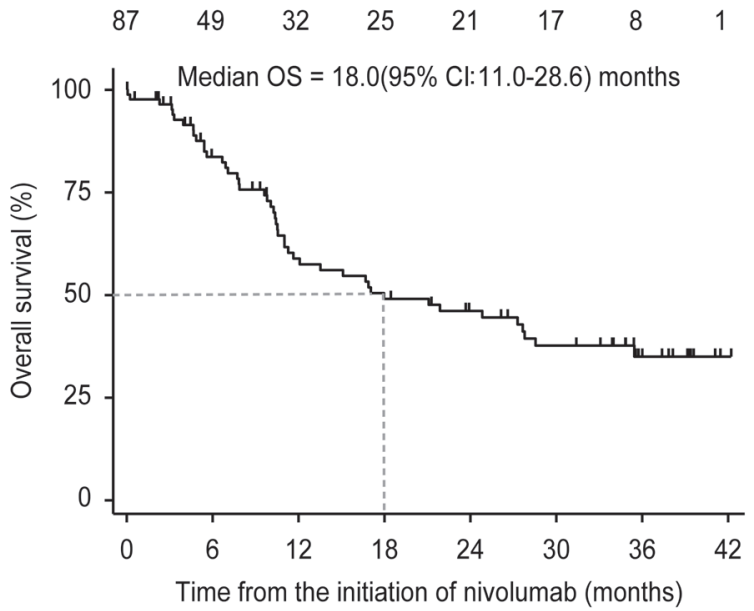

Number at risk

$$
\begin{array}{llllllll}
87 & 63 & 42 & 35 & 29 & 22 & 10 & 1
\end{array}
$$

Figure 1. Kaplan-Meier curves of progression-free survival (PFS) and overall survival (OS) $(\mathrm{n}=87)$.

to 15.1 (10.3-28.6) in patients with no therapy after the nivolumab (Table 3). Complete or partial response (ORR) was achieved by $25 / 81$ (31\%; $95 \%$ CI: $21 \%$ to $41 \%)$ patients, and complete or partial response or disease stabilization (DCR) was achieved by $43 / 81$ patients (53\%; $95 \%$ CI: $43 \%$ to $63 \%$ ) (Table 3). The median time to response was 4.7 ( $95 \%$ CI: 3.0 to 7.7 ) months, and in responders, the median duration of complete or partial response (ORR) was 21 months. In the bivariable unadjusted analysis, we observed a significant unfavorable association of bone metastasis and treatment-related and immune-mediated adverse events association with PFS (Table 4). Bone metastasis remained significantly unfavorably associated with PFS after the adjustment for age, sex, ECOG performance status at the time of nivolumab initiation, and the time from diagnosis to the introduction of nivolumab (adjusted $\mathrm{HR}=1.76 ; 95 \% \mathrm{CI}$ : $1.00-3.10 ; \mathrm{p}=0.049$; FDR).
Table 2 . Safety and tolerability $(n=87)$.

\begin{tabular}{|c|c|c|c|c|c|c|}
\hline & \multicolumn{3}{|c|}{ Any grade } & \multicolumn{3}{|c|}{ Grade 3-4 } \\
\hline & $\mathbf{n}$ & $(\%)$ & $(95 \% \mathrm{CI})$ & $\mathbf{n}$ & $(\%)$ & $(95 \% \mathrm{CI})$ \\
\hline $\begin{array}{l}\text { Treatment discontinuation } \\
\text { because of toxicity }\end{array}$ & 4 & (5) & $(1-12)$ & & & \\
\hline $\begin{array}{l}\text { Treatment-related adverse } \\
\text { events }\end{array}$ & 37 & (43) & $(32-54)$ & 4 & $(5)$ & $(0-10)$ \\
\hline $\begin{array}{l}\text { Treatment-related immune- } \\
\text { mediated adverse events }\end{array}$ & 22 & $(25)$ & $(17-35)$ & 4 & $(5)$ & $(0-10)$ \\
\hline \multicolumn{7}{|l|}{ All adverse events } \\
\hline dermatitis, rash, pruritus & 11 & (13) & $(6-20)$ & & & \\
\hline anemia & 6 & $(7)$ & $(2-12)$ & 2 & $(2)$ & $(0-5)$ \\
\hline fatigue & 5 & $(6)$ & $(1-11)$ & & & \\
\hline diarrhea & 5 & $(6)$ & $(1-11)$ & 1 & $(1)$ & $(0-3)$ \\
\hline hepatotoxicity & 5 & $(6)$ & $(1-11)$ & & & \\
\hline hypothyroidism & 4 & $(5)$ & $(0-10)$ & & & \\
\hline arthritis & 4 & (5) & $(0-10)$ & & & \\
\hline pain & 4 & $(5)$ & $(0-10)$ & & & \\
\hline nephropathy & 3 & (3) & $(0-7)$ & & & \\
\hline myositis & 2 & (2) & $(0-5)$ & & & \\
\hline dyspnea & 2 & $(2)$ & $(0-5)$ & & & \\
\hline swelling & 2 & $(2)$ & $(0-5)$ & & & \\
\hline xerostomia & 2 & $(2)$ & $(0-5)$ & & & \\
\hline gynecomastia & 2 & $(2)$ & $(0-5)$ & & & \\
\hline other, one patient ${ }^{\mathrm{b}}$ & & & & & & \\
\hline
\end{tabular}

Abbreviations: n-number of patients; CI-confidence interval; ${ }^{a}$ Data were missing for treatment discontinuation because of toxicity in $5(6 \%)$

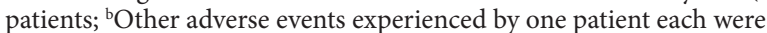
pneumonitis, fever, infection, decreased appetite, neuropathy, nausea, mild arthralgia, Guillain-Barré syndrome, erectile dysfunction, hearing loss, mucosal inflammation, ground glass opacities, hyperglycemia

Table 3. Efficacy $(n=87)$

\begin{tabular}{lccc}
\hline & $\mathbf{n}$ & $\mathbf{( \% )}$ & $\mathbf{( 9 5 \%} \mathbf{C I})$ \\
\hline Best response & & & \\
CR & 5 & $(6)$ & $(13-14)$ \\
PR & 20 & $(25)$ & $(16-35)$ \\
SD & 18 & $(22)$ & $(13-31)$ \\
PD & 26 & $(32)$ & $(22-42)$ \\
NA & 12 & $(15)$ & $(7-23)$ \\
ORR $^{a}$ & 25 & $(31)$ & $(21-41)$ \\
DCR $^{a}$ & 43 & $(53)$ & $(43-63)$
\end{tabular}

Time-to-event endpoints (months), median (95\% CI)

$\begin{array}{lcc}\text { TTR }^{\mathrm{a}} & 4.7 & (3.0-7.7) \\ \text { DOR }^{\mathrm{a}} & 21.2 & (4.1-\mathrm{NA}) \\ \text { PFS } & 8.5 & (4.9-12.1) \\ \text { OS } & 18.0 & (11.0-28.6)\end{array}$

OS stratified for therapy after nivolumab

therapy after $27.3 \quad$ (10.5-not reached) no therapy after $\quad 15.1 \quad(10.3-28.6)$

Abbreviations: CI-confidence interval; CR-complete response; PR-partial response; $\mathrm{SD}$-stable disease; $\mathrm{PD}$-progressive disease; NA-not evaluable; ORR-overall response rate; DCR-disease control rate; TTR-time to response; DOR-duration of response, PFS-progression free survival; OSoverall survival; ${ }^{a}$ Data were missing for best response, ORR and DCR in 6 (7\%) patients; for time to response and duration of response in $2 / 25$ (8\%) patients 
Table 4. Bivariable unadjusted association of patient characteristics with progression-free and overall survival ( $\mathrm{n}=\mathbf{8 7})$.

\begin{tabular}{|c|c|c|c|c|c|c|c|}
\hline & \multirow{2}{*}{$\mathbf{n}$} & \multicolumn{3}{|c|}{ PFS } & \multicolumn{3}{|c|}{ OS } \\
\hline & & HR & $(95 \% \mathrm{CI})$ & p-value & HR & $(95 \% \mathrm{CI})$ & p-value \\
\hline \multicolumn{8}{|c|}{ Age at nivolumab initiation } \\
\hline$\leq 64$ years & 51 & 1 & & & 1 & & \\
\hline$\geq 65$ years & 36 & 0.84 & $(0.50-1.41)$ & 0.517 & 0.81 & $(0.45-1.48)$ & 0.502 \\
\hline \multicolumn{8}{|l|}{ Sex } \\
\hline males & 69 & 1 & & & 1 & & \\
\hline females & 18 & 0.85 & $(0.45-1.60)$ & 0.620 & 1.01 & $(0.50-2.05)$ & 0.967 \\
\hline \multicolumn{8}{|c|}{ Metastatic sites } \\
\hline \multicolumn{8}{|l|}{ lung } \\
\hline no & 17 & 1 & & & 1 & & \\
\hline yes & 70 & 1.03 & $(0.54-1.99)$ & 0.919 & 1.48 & $(0.66-3.31)$ & 0.342 \\
\hline \multicolumn{8}{|c|}{ lymph nodes } \\
\hline no & 47 & 1 & & & 1 & & \\
\hline yes & 40 & 1.21 & $(0.75-2.00)$ & 0.471 & 0.97 & $(0.54-1.74)$ & 0.916 \\
\hline \multicolumn{8}{|l|}{ bone } \\
\hline no & 47 & 1 & & & 1 & & \\
\hline yes & 40 & 2.15 & $(1.28-3.62)$ & $0.004^{*}$ & 2.12 & $(1.18-3.80)$ & $0.012^{*}$ \\
\hline \multicolumn{8}{|c|}{ Number of metastatic sites } \\
\hline $1-2$ & 33 & 1 & & & 1 & & \\
\hline 3 & 29 & 1.61 & $(0.89-2.94)$ & 0.117 & 1.35 & $(0.69-2.62)$ & 0.377 \\
\hline$\geq 4$ & 25 & 1.52 & $(0.80-2.88)$ & 0.205 & 1.41 & $(0.66-2.99)$ & 0.372 \\
\hline \multicolumn{8}{|c|}{ ECOG performance status ${ }^{\mathrm{a}}$} \\
\hline 0 & 35 & 1 & & & 1 & & \\
\hline $1-2$ & 39 & 1.47 & $(0.86-2.52)$ & 0.156 & 2.08 & $(1.15-3.78)$ & $0.016^{*}$ \\
\hline \multicolumn{8}{|c|}{ Time from diagnosis to introduction of nivolumab } \\
\hline$\leq 1$ year & 30 & 1 & & & 1 & & \\
\hline $2-3$ years & 23 & 1.40 & $(0.76-2.59)$ & 0.278 & 1.38 & $(0.72-2.64)$ & 0.337 \\
\hline$\geq 4$ years & 34 & 0.59 & $(0.32-1.09)$ & 0.090 & 0.36 & $(0.16-0.78)$ & 0.010 \\
\hline \multicolumn{8}{|c|}{ Concomitant radiotherapy ${ }^{\mathrm{a}}$} \\
\hline no & 60 & 1 & & & 1 & & \\
\hline yes & 25 & 1.62 & $(0.95-2.75)$ & 0.074 & 1.59 & $(0.88-2.87)$ & 0.127 \\
\hline \multicolumn{8}{|c|}{ Treatment-related adverse events } \\
\hline no & 50 & 1 & & & 1 & & \\
\hline yes & 37 & 0.50 & $(0.29-0.84)$ & $0.010^{*}$ & 0.37 & $(0.20-0.69)$ & $0.002^{*}$ \\
\hline \multicolumn{8}{|c|}{ Treatment-related immune-mediated adverse events } \\
\hline no & 65 & 1 & & & 1 & & \\
\hline yes & 22 & 0.47 & $(0.25-0.87)$ & $0.016^{\star}$ & 0.31 & $(0.14-0.67)$ & $0.003^{*}$ \\
\hline
\end{tabular}

Abbreviation: PFS-progression-free survival; OS-overall survival; HR-hazard ratio; CI-confidence interval; p-statistical significance calculated using Cox proportional hazard regression, ${ }^{*}$ False discovery rate $<10 \%$; ${ }^{\text {DData }}$ were missing for ECOG performance status in 13 (15\%) patients; for concomitant radiotherapy in $2(2 \%)$ of patients

Both treatment-related and treatment-related immunemediated adverse events of any grade remained significantly associated with PFS after adjustment for the same four variables and the time-dependent-covariate number of nivolumab cycles $(\mathrm{HR}=0.51 ; 95 \% \mathrm{CI}$ : $0.27-0.93$; $\mathrm{p}=0.029 ; \mathrm{HR}=0.48 ; 95 \% \mathrm{CI}: 0.24-0.94 ; \mathrm{p}=0.032$, respectively).

OS was significantly unfavorably associated with bone metastasis and ECOG performance status 1-2 at the time of nivolumab introduction, but both associations were weakened and were not significant after adjusting for the mentioned covariates and the therapy after the discontinuation of nivolumab. OS was significantly favorably associated with $\geq 4$ years from diagnosis to the introduction of nivolumab, but this association was not significant after the adjustment for the mentioned covariates. The associations of OS with the treatment-related adverse events of any grade analyzed separately and treatment-related immunemediated adverse events of any grade were significant in the bivariable analysis (Table 4); the associations even increased and remained significant after adjusting for age at the initiation of nivolumab, sex, number of metastatic 
sites, ECOG performance status, time from diagnosis to the introduction of nivolumab, therapy after the discontinuation of nivolumab, number of cycles and concomitant radiotherapy ( $\mathrm{HR}=0.29 ; 95 \% \mathrm{CI}$ : $0.11-0.72 ; \mathrm{p}=0.008$; FDR $<10 \%$; HR $=12$; $95 \%$ CI: 0.04-037; $<<0.001$; FDR $<10 \%$ ).

\section{Discussion}

The results of this retrospective analysis of the nivolumab expanded-access program dataset provided evidence of the safety and effectiveness of nivolumab in real-world patients receiving treatment for metastatic RCC in Croatia, Hungary, and Malta, countries with some of the worst cancer outcomes in the European Union [21]. The clinical outcomes in the Croatian, Hungarian, and Maltese mRCC populations closely mirrored those obtained in the pivotal phase III randomized clinical trial of nivolumab for the second-line treatment of metastatic RCC [15]. Such a performance of a new drug in everyday practice is not always the case. Generally, discrepancies in new drug efficacy and toxicity, between the results from registrational trials and the results observed in everyday clinical practice, could exist due to differences in patient selection, organizational specificities, multidisciplinary use, and general level of oncological care [22-24]. Consequently, the results from randomized phase III trials are often difficult to repeat in general clinical practice, especially in the health systems where significant challenges in delivering optimal cancer care exist [24].

As in the real-world Italian study, we found a lower incidence of grade 3 or 4 treatment-related adverse events than the blinded, randomized controlled, multicenter phase III Checkmate 025 trial $[15,25]$, but the same incidence of grade 3 or 4 adverse events as in subgroup analysis of blinded, randomized phase II trial conducted in United States, Canada, Finland, and Italy [26]. This could be potentially explained by a difference in the process of obtaining information on adverse events in the clinical study setup and everyday practice where these adverse events may not be regularly registered, especially the ones with lower intensity. Compared to the patients in the Italian study, the patients in our study experienced somewhat more treatment-related adverse events of any grade but a somewhat lower rate of grade 3 or 4 adverse events and almost the same treatment discontinuation rate due to toxicity. Neither of the two studies recorded a toxicity-related death. While PFS was longer in our study, the median OS was similar in both studies. Again, in everyday practice, the PFS is a much less precise endpoint based on differences in followup procedures, their frequency, type, and quality. On the other hand, OS is a strong endpoint that is equally good in everyday practice and in clinical trials, especially for patients with mRCC at that time and in that region, where less further therapies were available to prolong post-progression survival. In our study, only 19/80 (24\%) patients received post-progression therapy, but we included it in the multivariable analysis as a covariate and controlled its effects that way. A very important concordant result between our two studies was the finding of a favorable prognostic value in terms of treatment-related immunemediated adverse events. This favorable association of immune-related adverse events with prolonged survival had previously been shown in the treatment of melanoma and non-small-cell lung cancer, and some opposite results were presented as well; however, this subject has not been studied enough [27-29]. Therefore, it is important that we confirm these findings using a different method to control the possible confounders and a different selection of covariates. The problem of a seemingly paradoxical beneficial prognostic value of immune-related adverse events should be addressed by a prospective study using an endpoint that can be measured before and after the occurrence of the adverse event. This association may partially be explained by the on-target toxicity or the activation of the immune system that is not only directed at the tumor but also at the general tissue. A similar OS (20.6 months after the exclusion of patients with non-clear cell vs. 18 months in our study) was found in the US-based study of real-world data of mRCC patients treated with nivolumab from the International Metastatic Renal Cell Carcinoma Database Consortium, reflecting the applicability of the drug in regions and countries with developed oncology infrastructures [30]. In our study, we have defined efficacy and safety of nivolumab similar to these obtained from the registrational trial as well as from real-world data from developed countries in the region where significant challenges in delivering optimal cancer control exist. Consequently, we can conclude that nivolumab outcomes in the therapy of mRCC are not a system- but patient- and tumor-based.

The main limitation of our study was the high risk of unmeasured confounding effects and bias. As this was a real-world, observational, retrospective study, we could not use a randomized control group to control for all possible confounders. However, our study results were in line with the randomized controlled phase III study that was performed on a comparable population as well as other studies conducted in different real-world patient scenarios. We used electronic medical records as the source of study data, and although we carefully checked each record, we could not control the quality of the original entries. As this was a retrospective study, all treatment decisions were made long before study initiation and we had no control over their specifics. The main strength of our study is its better external validity due to the unselected general population of patients diagnosed with $\mathrm{mRCC}$ and treated with nivolumab as $\mathrm{a} \geq 2^{\text {nd }}$ line treatment.

In conclusion, nivolumab is acceptably safe, tolerable, and effective in the real-world treatment of previously treated mRCC. 


\section{References}

[1] MOTZER RJ, HUTSON TE, TOMCZAK P, MICHAELSON MD, BUKOWSKI RM et al. Sunitinib versus interferon alfa in metastatic renal-cell carcinoma. N Engl J Med 2007; 356: 115-124. https://doi.org/10.1056/NEJMoa065044

[2] MOTZER RJ, HUTSON TE, CELlA D, REEVES J, HAWKINS R et al. Pazopanib versus sunitinib in metastatic renal-cell carcinoma. N Engl J Med 2013; 369: 722-731. https://doi.org/10.1056/NEJMoa1303989

[3] MOTZER RJ, NOSOV D, EISEN T, BONDARENKO I, LESOVOY $\mathrm{V}$ et al. Tivozanib versus sorafenib as initial targeted therapy for patients with metastatic renal cell carcinoma: results from a phase III trial. J Clin Oncol 2013; 31: 3791-3799. https://doi.org/10.1200/jco.2012.47.4940

[4] HUDES G, CARDUCCI M, TOMCZAK P, DUTCHER J, FIGLIN R et al. Temsirolimus, interferon alfa, or both for advanced renal-cell carcinoma. N Engl J Med 2007; 356: 2271-2281. https://doi.org/10.1056/NEJMoa066838

[5] CHOUEIRI TK, HESSEL C, HALABI S, SANFORD B, MICHAELSON MD et al. Cabozantinib versus sunitinib as initial therapy for metastatic renal cell carcinoma of intermediate or poor risk (Alliance A031203 CABOSUN randomised trial): progression-free survival by independent review and overall survival update. Eur J Cancer 2018; 94: 115-125. https://doi.org/10.1016/j.ejca.2018.02.012

[6] RINI BI, ESCUDIER B, TOMCZAK P, KAPRIN A, SZCZYLIK $C$ et al. Comparative effectiveness of axitinib versus sorafenib in advanced renal cell carcinoma (AXIS): a randomised phase 3 trial. Lancet 2010; 378: 1931-1939. https:// doi.org/10.1016/s0140-6736(11)61613-9

[7] MOTZER RJ, ESCUDIER B, OUDARD S, HUTSON TE, PORTA $C$ et al. Efficacy of everolimus in advanced renal cell carcinoma: a double-blind, randomised, placebo-controlled phase III trial. Lancet 2008; 372: 449-456. https://doi. org/10.1016/s0140-6736(08)61039-9

[8] MOTZER RJ, BACIK J, MURPHY BA, RUSSO P, MAZUMDAR M. Interferon-alfa as a comparative treatment for clinical trials of new therapies against advanced renal cell carcinoma. J Clin Oncol 2002; 20: 289-296. https://doi. org/10.1200/jco.2002.20.1.289

[9] DRAKE CG, JAFFEE E, PARDOLL DM. Mechanisms of immune evasion by tumors. Adv Immunol 2006; 90: 51-81. https://doi.org/10.1016/s0065-2776(06)90002-9

[10] ZITVOGEL L, TESNIERE A, KROEMER G. Cancer despite immunosurveillance: immunoselection and immunosubversion. Nat Rev Immunol 2006; 6: 715-727. https://doi. org/10.1038/nri1936

[11] HAMID O, CARVAJAL RD. Anti-programmed death-1 and anti-programmed death-ligand 1 antibodies in cancer therapy. Expert Opin Biol Ther 2013; 13: 847-861. https://doi.org /10.1517/14712598.2013.770836

[12] HARGADON KM, JOHNSON CE, WILLIAMS CJ. Immune checkpoint blockade therapy for cancer: an overview of FDA-approved immune checkpoint inhibitors. Int Immunopharmacol 2018; 62: S29-39. https://doi.org/10.1016/j. intimp.2018.06.001
[13] TOPALIAN SL, HOD FS, BRAHMER JR, GETTINGER SN, SMITH DC et al. Safety, activity, and immune correlates of anti-PD-1 antibody in cancer. N Engl J Med 2012; 366: $2443-$ 2454. https://doi.org/10.1056/NEJMoa1200690

[14] FAROLFI A, SCHEPISI G, CONTEDUCA V, BURGIO SL, LOLLI $C$ et al. Pharmacokinetics, pharmacodynamics and clinical efficacy of nivolumab in the treatment of metastatic renal cell carcinoma. Expert Opin Drug Metab Toxicol 2016; 12: 1089-1096. https://doi.org/10.1080/17425255.2016.1214 713

[15] MOTZER RJ, ESCUDIER B, MCDERMOTT DF, GEORGE $S$, HAMMERS $\mathrm{H}$ et al. Nivolumab versus everolimus in advanced renal-cell carcinoma. N Engl J Med 2015; 373: 1803 1813. https://doi.org/10.1056/NEJMoa1510665

[16] CURADO MP, EDWARDS B, SHIN HR, STORM H, FERLAY J et al. Cancer incidence in five continents. International Agency for Research in Cancer, Lyon 2007

[17] LEVI F, LUCCHINI F, NEGRI E, BOYLE P, LA VECCHIA C. Cancer mortality in Europe, 1995-1999, and an overview of trends since 1960. Int J Cancer 2004; 110: 155-169. https:// doi.org/10.1002/ijc.20097

[18] LEVI F, LUCCHINI F, NEGRI E, ZATONSKI W, BOYLE P et al. Trends in cancer mortality in the European Union and accession countries, 1980-2000. Ann Oncol 2004; 15: 14251431. https://doi.org/10.1093/annonc/mdh346

[19] BERRINO F, DE ANGELIS R, SANT M, ROSSO S, BIELSKA-LASOTA $M$ et al. Survival for eight major cancers and all cancers combined for European adults diagnosed in 1995-99: results of the EUROCARE-4 study. Lancet Oncol 2007; 8: 773-783. https://doi.org/10.1016/s14702045(07)70245-0

[20] WORLD MEDICAL ASSOCIATION. World Medical Association declaration of Helsinki: ethical principles for medical research involving human subjects. JAMA 2013; 310: 21912194. https://doi.org/10.1001/jama.2013.281053

[21] ALLEMANI C, MATSUDA T, DI CARLO V, HAREWOOD $\mathrm{R}$, MATZ $\mathrm{M}$ et al. Global surveillance of trends in cancer survival 2000-14 (CONCORD-3): analysis of individual records for 37513025 patients diagnosed with one of 18 cancers from 322 population-based registries in 71 countries. Lancet 2018; 391: 1023-1075. https://doi.org/10.1016/s01406736(17)33326-3

[22] TEMPLETON AJ, VERA-BADILLO FE, WANG L, ATTALLA M, DE GOUVEIA P et al. Translating clinical trials to clinical practice: outcomes of men with metastatic castration resistant prostate cancer treated with docetaxel and prednisone in and out of clinical trials. Ann Oncol 2013; 24: 2972-2977. https://doi.org/10.1093/annonc/mdt397

[23] SARGENT D. What constitutes reasonable evidence of efficacy and effectiveness to guide oncology treatment decisions? Oncologist 2010; 15: 19-23. https://doi.org/10.1634/ theoncologist.2010-S1-19

[24] GEORGE SL. Reducing patient eligibility criteria in cancer clinical trials. J Clin Oncol 1996; 14: 1364-1370. https://doi. org/10.1200/jco.1996.14.4.1364 
[25] VERZONI E, CARTENI G, CORTESI E, GIANNARELLI D, DE GIGLIO A et al. Real-world efficacy and safety of nivolumab in previously-treated metastatic renal cell carcino$\mathrm{ma}$, and association between immune-related adverse events and survival: the Italian expanded access program. J Immunother Cancer 2019; 7: 99. https://doi.org/10.1186/s40425019-0579-z

[26] GEORGE S, MOTZER RJ, HAMMERS HJ, REDMAN BG, KUZEL TM et al. Safety and Efficacy of Nivolumab in Patients With Metastatic Renal Cell Carcinoma Treated Beyond Progression: A Subgroup Analysis of a Randomized Clinical Trial. JAMA Oncol. 2016; 2: 1179-86. https://doi. org/10.1001/jamaoncol.2016.0775

[27] DAS S, JOHNSON DB. Immune-related adverse events and anti-tumor efficacy of immune checkpoint inhibitors. J Immunother Cancer 2019; 7: 306. https://doi.org/10.1186/ s40425-019-0805-8
[28] ATTIA P, PHAN GQ, MAKER AV, ROBINSON MR, QUEZADO MM et al. Autoimmunity correlates with tumor regression in patients with metastatic melanoma treated with anti-cytotoxic T-lymphocyte antigen-4. J Clin Oncol 2005; 23: 6043-6053. https://doi.org/10.1200/jco.2005.06.205

[29] TOI Y, SUGAWARA S, KAWASHIMA Y, AIBA T, KAWANA $S$ et al. Association of immune-related adverse events with clinical benefit in patients with advanced non-small-cell lung cancer treated with nivolumab. Oncologist 2018; 23: 13581365. https://doi.org/10.1634/theoncologist.2017-0384

[30] STUKALIN I, WELLS JC, GRAHAM J, YUASA T, BEUSELINCK B et al. Real-world outcomes of nivolumab and cabozantinib in metastatic renal cell carcinoma: results from the international metastatic renal cell carcinoma database consortium. Curr Oncol 2019; 26:e175-e179. https:// doi.org/10.3747/co.26.4595 